\title{
Behavioral assessments for pre-clinical pharmacovigilance
}

\section{James H. Thompson ${ }^{\dagger}$ and Teresa L. Micheli*t}

Behavioral Science Division, Clever Sys, Inc., Reston, VA, USA

*Correspondence: tmicheli@cleversysinc.com

${ }^{+}$James H. Thompson and Teresa L. Micheli contributed equally to this work.

Want to try a fun social experiment? Mention the term behavioral research to any scientist and watch their reaction. Some find the idea of behavioral research daunting, while others think the field isn't really a science, just the mere act of watching a subject's performance while making a few checkmarks on a clipboard. For those that have run behavioral experiments, we understand the theory and importance behind the research, and have an appreciation for a thorough and coherent experimental study.

In the last decade, the field of Neuroscience has been more accepting of behavior as a necessary part of the empirical studies that previously contained only cellular or histological findings. Science in general, is moving toward translational research (Zerhouni, 2005), so being able to show how the cellular or chemical changes affect the organism as a whole, has become a high priority. Transgenic mice have contributed enormous amounts of data to every sub category of neuroscience. The great minds of Biomedical Scientists are able to splice cells, taking out genes or introducing new ones in, but they need the expertise of a Behavioral Scientist in order to assess the changes to the organism that can not tell us what it is thinking or feeling.

So let's explore this further and start by asking, where does behavioral testing begin, and why is it important? The answers to both depend on the study you are conducting, especially when you are considering adding a substance into the mix that can alter physiology. Genetically mutated mice are engineered to determine the effects of a specific gene, or more commonly, in an effort to mimic human symptoms of disease. Pharmaceutical companies and toxicologists find themselves needing to comprehensively assess drug efficacy in a high throughput fashion, something that can take years to do in both human and non-human primates over the course of the lifespan. The development of the transgenic mouse, allows pre-clinical screening, on a mass level in a comparatively short period of time. This has created a major concern for drug research studies in terms of accurately determining what changes can be attributed to the drug as opposed to the changes in behavior due to the transgene manipulation. Therefore, prior to using any transgenic mouse model of disease, the researcher needs to know what changes the gene modification has caused in the mouse model, and places a great emphasis on the art of behavioral phenotyping. If you know what behaviors are "normal" for your mouse model, then you can determine what tests to use in a battery of assessments, including what home cage behaviors are important in your selection of data points. Take a current Alzheimer's disease (AD) model as an example, the $\mathrm{Tg} 2576$ for which the background strain is C57BL/6J. This particular $\mathrm{AD}$ mouse model carries the human gene for amyloid precursor protein (APP), which is responsible for the formation of $\mathrm{AD}$ like plaques in humans (Hsiao et al., 1996). Bred from a mouse that has the recessive gene $P d e b^{r d l}$, severe visual impairments (Gimenez and Montoliu, 2001) and deafness (Johnson et al., 1997) are common in these animals after 9 months of age. Additionally, after a period of time the animals begin to lose their hair and whiskers, and barber (over groom) themselves. It is necessary that these key characteristics are known prior to designing/conducting any behavioral task that uses auditory or visual cues for the animal to complete, as results might imply cognitive deficits.

\section{HOME CAGE BEHAVIORS}

Home cage behaviors in laboratory animal research are important because they are the best measure scientist's have, of the natural occurring behavior of the animal being studied. Since testing occurs in the animal's home environment, there are no confounds associated with human interference, testing protocol, or behavioral changes associated with moving the animal to another testing apparatus, just basic naturalistic observations of normal laboratory animal behavior. Typical home cage behavior monitoring includes measurements of circadian rhythms in addition to locomotor and stereotypic (pathologically repetitive) behaviors, all of which will be changed if a pharmaceutical intervention has an adverse effect on the animal or if the genetic mutation has changed the mouse model (Bordone et al., 2007; Dickinson et al., 2009; Jackson et al., 2009). Excessive grooming is a good example of why you would want to identify the phenotypic behaviors of your model prior to drug treatment. A pharmaceutical company may interpret barbering behaviors in the $\mathrm{Tg} 2576$ model as skin sensitivity due to the treatment, when in fact it is merely a typical characteristic for this mouse model. Therefore, not only can you obtain information about phenotypic behaviors by including home cage analysis in a study, you're also capable of collecting data about drug efficacy.

Many are discouraged from including home cage analysis within a study due to the time constraints of monitoring cages $24 / 7$, not to mention the complexity of numerous cages. However, just as the technology has assisted with the GLP compliance issues, it can also assist with animal testing issues. Roughan et al. (2009) used an automated behavioral recognition program to monitor pain and analgesic effects in mice while in the home cage after surgery. Results suggest that the program was as good as human monitoring. The program also provided investigators measurements that would have been otherwise impossible for a human scorer such as distance traveled throughout the cages and durations of all the various events. In a Huntington's disease mouse model, Steele et al. (2007) found that an automated home cage analysis found significant differences between $\mathrm{Tg}$ and Wt mice 6 weeks prior to the onset of the disease. Clinical phenotypes of human Huntington's such 
as the rest-wake abnormalities, not previously identified in a mouse model, was also observed using the automated system. The inclusion of automated high throughput technology in lieu of human observation makes this monitoring as efficient as possible. Identifying subtle phenotypic behavioral changes early in experimentation, and ones closely related to human behaviors, would streamline the pre-clinical drug efficacy process and increase pre-clinical pharmacovigiliance.

\section{BEHAVIORAL BATTERIES}

Once the mouse model has been properly phenotyped and any drug or genetic effects ascertained via home cage observation, neurobehavioral assessments must be completed. In very specified studies, one or two tests that target a particular brain region for assessment may be enough. However in most high throughput toxicology and drug studies, it is more important to do an overall assessment, to determine all impairments or enhancements due to treatment, drug or otherwise. Perturbations in sensory processing resulting from genetic alterations or drug effects may not be identified from just a couple of tasks, leading researchers to misinterpret the assessments. If a particular test relies on visual inputs for instance, such as the radial arm maze, and the model has poor visual acuity, then learning and memory may appear impaired (Garcia et al., 2004). If a behavioral battery were to be utilized in this case, learning and memory deficits might not be diagnosed, as results from other tasks such as olfactory discrimination would not demonstrate cognitive deficits. A comprehensive battery of tests is a recommended procedure to accurately determine the behavioral profile of a particular mouse model in any drug study. Not only is a battery of tests suggested, but also the pre-clinical phase should examine early effects, long-term effects, and lifespan effects of the treatment. These data would be the most pertinent in studies being translated into clinical models of the same design. In the long run, these behavioral studies at the pre-clinical stage will ensure that drugs progress to clinical trials in the fastest, safest, and most effective manner possible. Though the inclusion of more data may seem to lengthen studies, it would strengthen safety. While many scientists look at CNS disorders and drugs from a cellular level, we are dealing with diseases and side effects that manifest themselves behaviorally. Therefore, it is imperative that we look at them from a behavioral standpoint, and conduct research in a responsible and inclusive manner when it comes to drug development.

\section{CONCLUSION}

Using an automated home cage behavioral assessment to identify any adverse effects, early and throughout treatment, would be cost effective and efficient for drug companies. Overall, home cage behavioral measures, along with toxicology reports, behavioral assessments, and histology, gives researchers the most comprehensive information for pre-clinical screening. For the toxicologist this means more accurately targeted molecules, and mass data points for preliminary screening; for nonresearchers, this means less danger to the participants in clinical trials, and safer more effective drugs delivered to the public more efficiently. Stringent pre-clinical measures over the rodents' lifespan (approximately 2 years), would increase pre-clinical pharmacovigilance, and decrease potential adverse drug effects to the human population (Maennl, 2008).

\section{REFERENCES}

Bordone, L., Cohen, D., Robinson, A., Motta, M. C., van Veen, E., Czopik, A., Steele, D. A., Crowe, H., Marmor, S., Luo, J., Gu, W., and Guarente, L. (2007). SIRT1 transgenic mice show phenotypes resembling calorie restriction. Aging Cell 6, 759-767.

Dickinson, A. L., Leach, M. C., and Flecknell, P.A. (2009). The analgesic effects of oral paracetamol in two strains of mice undergoing vasectomy. Lab. Anim. 43, 357-361.

Garcia, M., Gordon, M., Hutton, M., Lewis, J., McGowan, E., Dickey, C., Morgan, D., and Arendash, G.W. (2004). Retinal degeneration (rd) gene seriously impairs spatial cognitive performance in normal and Alzheimer's transgenic mice. Neuroreport 15, 73-77.

Gimenez, E., and Montoliu, L. (2001). A simple polymerase chain reaction assay for genotyping the retinal degeneration mutation (Pdebrd1) in FVB/N-derived transgenic mice. Lab. Anim. 35, 153-156.

Hsiao, K., Chapman, P., Nilsen, S., Eckman, C., Harigaya, Y., Younkin, S., Yang, F., and Cole, G. (1996). Correlative memory deficits, Abeta elevation, and amyloid plaques in transgenic mice. Science 274, 99-102.

Jackson, W. S., Borkowski, A. W., Fass, H., Steele, A. D., King, O. D., Watson, N., Jasanoff, A., and Lindquist, S. (2009). Spontaneous generation of prion infectivity in fatal familial insomnia knockin mice. Neuron $63,438-450$.

Johnson, K. R., Erway, L. C., Cook, S. A., Willott, J. F., and Qing,Y.Z. (1997).A major gene affecting age-relationed hearing loss in C57BL/6J mice. Hear. Res. 114, 83-92.

Maennl, U. (2008). Pharmacovigilance: a company-wide challenge. Appl. Clin. Trials 17, 50-58.

Roughan, J.V., Wright-Williams, S. L., and Flecknell, P.A. (2009). Automated analysis of postoperative behavior: assessment of HomeCageScan as a novel method to rapidly identify pain and analgesic effects in mice. Lab. Anim. 43, 17-26.

Steele, A. D., Jackson, W. S., King, O. D., and Lindquist, S. (2007). The power of automated high-resolution behavior analysis revealed by its application to mouse models of Huntington's and prion diseases. PNAS 104, 1983-1988.

Zerhouni, E. A. (2005). Translational and clinical sciencetime for a new vision. N. Engl. J. Med. 353, 1621-1623.

Received: 02 December 2009; accepted: 01 March 2010; published online: 22 March 2010.

Citation: Thompson JH and Micheli TL (2010) Behavioral assessments for pre-clinical pharmacovigilance. Front. Neurosci. 4:27. doi: 10.3389/fnpha.2010.00003

Thisarticlewassubmitted to Frontiers in Neuropharmacology, a specialty of Frontiers in Neuroscience.

Copyright (c) 2010 Thompson and Micheli. This is an open-access article subject to an exclusive license agreement between the authors and the Frontiers Research Foundation, which permits unrestricted use, distribution, and reproduction in any medium, provided the original authors and source are credited. 